\title{
High sensitivity humidity sensor based on cladding-etched optical fiber and lossy mode resonances
}

\author{
J. Ascorbe*, J.M.Corres, I.R. Matias, F.J Arregui \\ Institute of Smart Cities, Public University of Navarra \\ Pamplona, Spain \\ *joaquin.ascorbe@unavarra.es
}

Keywords: Lossy Mode Resonances; Cladding-etched single mode optical fiber; Optical fiber humidity sensor; Tin oxide; Thin films

\begin{abstract}
In this work a high sensitivity optical fiber humidity sensor (OFHS) is presented. The configuration chosen for this purpose is a cladding-etched single mode optical fiber (CE-SMF) coated with a thin film of tin oxide $\left(\mathrm{SnO}_{2}\right)$. The etching has been made using hydrofluoric acid (HF) and the coating has been fabricated by means of sputtering. Tin oxide was used to build the nano-coating which produces the Lossy Mode Resonance (LMR) and works as sensitive material. Theoretical and experimental results are shown and compared. The device was tested using a climatic chamber in order to obtain the response of the OFHS to relative humidity. Changes greater than $130 \mathrm{~nm}$ have been obtained for relative humidity varying from $20 \%$ to $90 \%$, which gives a sensitivity of $1.9 \mathrm{~nm} / \% \mathrm{RH}$.
\end{abstract}

\section{Introduction}

Optical fiber humidity sensors (OFHS) offer several advantages over electronic humidity sensor like small size, lightness, the possibility of working on flammable environments and, the most important, their electromagnetic immunity. Other important advantage of OFHS over electronic humidity sensor is the possibility of working at higher temperature and pressure ranges.

It can be distinguished different ways of measuring relative humidity with an OFHS. The first way would be by amplitude based techniques which consist of measuring changes in the transmitted optical power caused by changes on the optical absorption or on the refractive index of the coating $[1,2,3,4]$. The other way is named wavelength based techniques, which overcome the limitations of the previous technique, such as external noises or power fluctuations [5]. Other techniques include measuring shifts on the phase of the light or measuring the state of polarization, but these methods require more complex setups.

For wavelength based techniques, transmission bands or absorption peaks in the transmission profile should be achieved in order to measure changes on the wavelength where that absorption peak is placed. Absorption peaks could be achieved using several structures, such as long period gratings [6], photonic crystal fibers [7] or interferometric structures [8]; and transmission bands could be achieved, for example, using a single mode-multimode-single mode structure [9]. Most of these structures generate an absorption peak at certain wavelength by creating a grating on the core of the optical fiber, modifying its refractive index or with interferometers. These structures present some desirable characteristics, such as small spectral width of the attenuation band. On the other hand, the maximum wavelength shift of these 
devices is around $5 \mathrm{~nm}[7,8]$. Another method for achieving an absorption peak is the generation of Lossy Mode Resonances (LMRs).

LMRs are electromagnetic resonances that are generated by some materials deposited over a waveguide. Then, coupling of light to the cladding modes happens at certain resonance wavelengths, without modifying the refractive index of the core, provided that the material meets the requirements for its generation. This condition is that the real part of the thin-film permittivity is positive and higher in magnitude than both its own imaginary part and the real part permittivity of the materials surrounding the thin-film [10]. There is a wide variety of materials that can be used to generate LMRs, e.g., polymers and semiconductors, allowing fabricating optical fiber sensor for multiple applications [10-17]. Generation of LMRs on single mode optical fiber (SMF) has generally been done with tapered optical fibers $[17,19]$ as the way to get access to the evanescent field. In this work a cladding-etched single mode optical fiber (CE-SMF) coated with a nano-coating has been used for the fabrication of the OFHS. Etching the optical fiber has some advantages over conventional tapered optical fibers, like providing a much more repetitive process from the point of view of the physical parameters of the resultant taper. The main difference between tapered and cladding etched optical fiber is that in the first case both the cladding and the core are narrowed, maintaining the relation core/cladding, whereas in the second case only the diameter of the cladding is reduced [20-22]. Development of adiabatic tapered optical fibered by means of chemical etching has been previously studied $[20,21,23]$ and some theoretical approaches can be seen in [24]. There are also some OFHS that have employed chemical etching, for example, to improve the sensitivity of some interferometric devices [6], or to taper a plastic optical fiber and then, by adding an appropriate coating $[3,4]$, to develop an evanescent wave OFHS, generally based on changes on the optical absorption or the refractive index of the coating which leads to changes on the transmitted optical power. Chemical etching has been also applied to other structures as long period gratings optical fibers in order to improve their sensitivity [25]. Here, a nano-coating has been coated onto the CE-SMF in order to achieve the LMR which allows to measure changes in the wavelength where the LMR occurs.

The material that has been chosen for the fabrication of the nano-coating is tin oxide $\left(\mathrm{SnO}_{2}\right)$. The etched optical fiber was coated with this material by means of sputtering because it is a method that provides homogeneous films with good control of the thickness deposited. Tin oxide has a relatively high refractive index; it is sensitive to changes on the relative humidity and besides it meets the requirements for generating LMRs. Several materials have been previously studied as LMR generators and as humidity sensors, most of them coating a plastic cladding removed optical fiber $(200 \mu \mathrm{m})$, such as PAH/PAA [26], ITO [27], $\ln _{2} \mathrm{O}_{3}[29,30], \mathrm{TiO}_{2} / \mathrm{PSS}$ [13] and $\mathrm{SnO}_{2}$ [30] etc. obtaining the best sensitivity for [13], with values of $0.4 \mathrm{~nm} / \% \mathrm{RH}, 0.833 \mathrm{~nm} / \% \mathrm{RH}$, $0.133 \mathrm{~nm} / \% \mathrm{RH}, 0.935 \mathrm{~nm} / \% \mathrm{RH}, 1.43 \mathrm{~nm} / \% \mathrm{RH}$ and $0.107 \mathrm{~nm} / \mathrm{RH}$ respectively. Devices developed in previous works have been fabricated by dip-coating and a post-annealing process or by Layer-by-Layer assembly, which are slow methods and they also present some dependence on the environmental conditions. Furthermore, polymeric coatings present greater hysteresis and higher response times than the device developed here.

The device developed in this work improves the sensitivity of previous devices [6-8, 13, 26-30], it is relatively simple to fabricate and it has a smaller FWHM than other devices based on LMRs $[17,26]$. Besides it has short response times, good linearity and low hysteresis. To our knowledge, this is the first time that a cladding-etched single mode fiber is used for the fabrication of a Lossy Mode Resonance sensor. The manuscript is organized as follows: firstly, in 
section II, the structure, the fabrication of the device and the experimental set-up are analyzed. In section III the theoretical results, obtained with FIMMWAVE ${ }^{\circledR}$ software, are shown and explained. In section IV the device performance is discussed, and finally some concluding remarks are exposed in Section V.

\section{Device Fabrication}

\section{A. Chemical etching}

For the chemical etching, hydrofluoric acid (HF) diluted at 40\% (Sigma-Aldrich) was used. Then the SMF $(9 / 125 \mu \mathrm{m}$, core/cladding diameter) purchased from Telnet Rl, with $5 \mathrm{~mm}$ of the buffer removed, was immersed in the HF. When the external diameter reaches $60 \mu \mathrm{m}$, the etched SMF was washed with water to remove all the HF. Next step is to immerse the SMF in $20 \%$ diluted HF to have fine control on the etching process until the external diameter reaches $19 \mu \mathrm{m}$. This diameter has been proved to be good enough for getting access to the evanescent field and obtaining the LMR. Finally it was washed again with water to remove the HF and it was attached to a U-holder to keep the fiber straight for next steps. The cladding-etched optical fiber can be seen in Fig. 1.

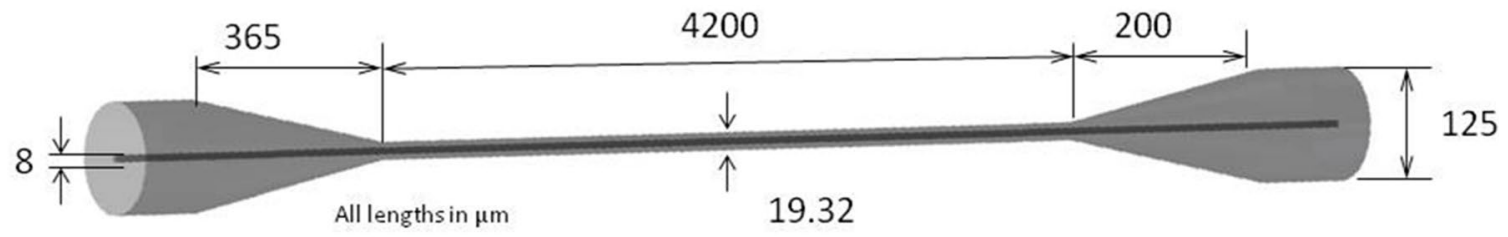

Fig. 1.- Cladding-etched single mode optical fiber.

\section{B. Sputtering Coating}

The CE-SMF was introduced in the vacuum chamber of the sputtering machine, which was a Pulsed DC-Sputtering System (Nadetech Inc.) and coated with tin oxide $\left(\mathrm{SnO}_{2}\right)$. The $\mathrm{SnO}_{2}$ target, 99.99\% of purity, was purchased from ZhongNuo Advanced Material Technology Co. The sputtering process was done at 9×10-3 mbar and $180 \mathrm{~mA}$ and when half of the time has been completed ( 6 minutes), it has been turned 180 and coated for other 6 minutes. The thickness of the coating has been estimated by means of an AFM (Veeco Innova model 840-012-711) to be $140 \mathrm{~nm}$ for device D1. This thickness causes a LMR located at $1550 \mathrm{~nm}$. If the thickness of the $\mathrm{SnO}_{2}$ decreases the $\mathrm{LMR}$ shifts to lower wavelengths due to its sensitiveness to the surrounding medium refractive index $[5,19]$. On the other hand, if the thickness increases the LMR shifts to greater wavelengths until disappear, and other LMR will appear. Second LMR is less sensitive than the first, and so on. That will be demonstrated by developing other devices, D2 to D4, coated by a DC Sputtering System (Quorum K750X) at $9 \times 10^{-2}$ mbar and $90 \mathrm{~mA}$. Thickness of device D2 is $275 \mathrm{~nm}$, thickness of device D3 is $250 \mathrm{~nm}$, while D4 has a coating thickness of 650 $\mathrm{nm}$, which means that the third LMR has appeared. In Fig.2 it is shown the refractive index of the coating which has been measured by ellipsometry (Uvisel 2, Horiba). For the wavelengths where the device works the coating has a refractive index of 1.92 and a low extinction coefficient. 


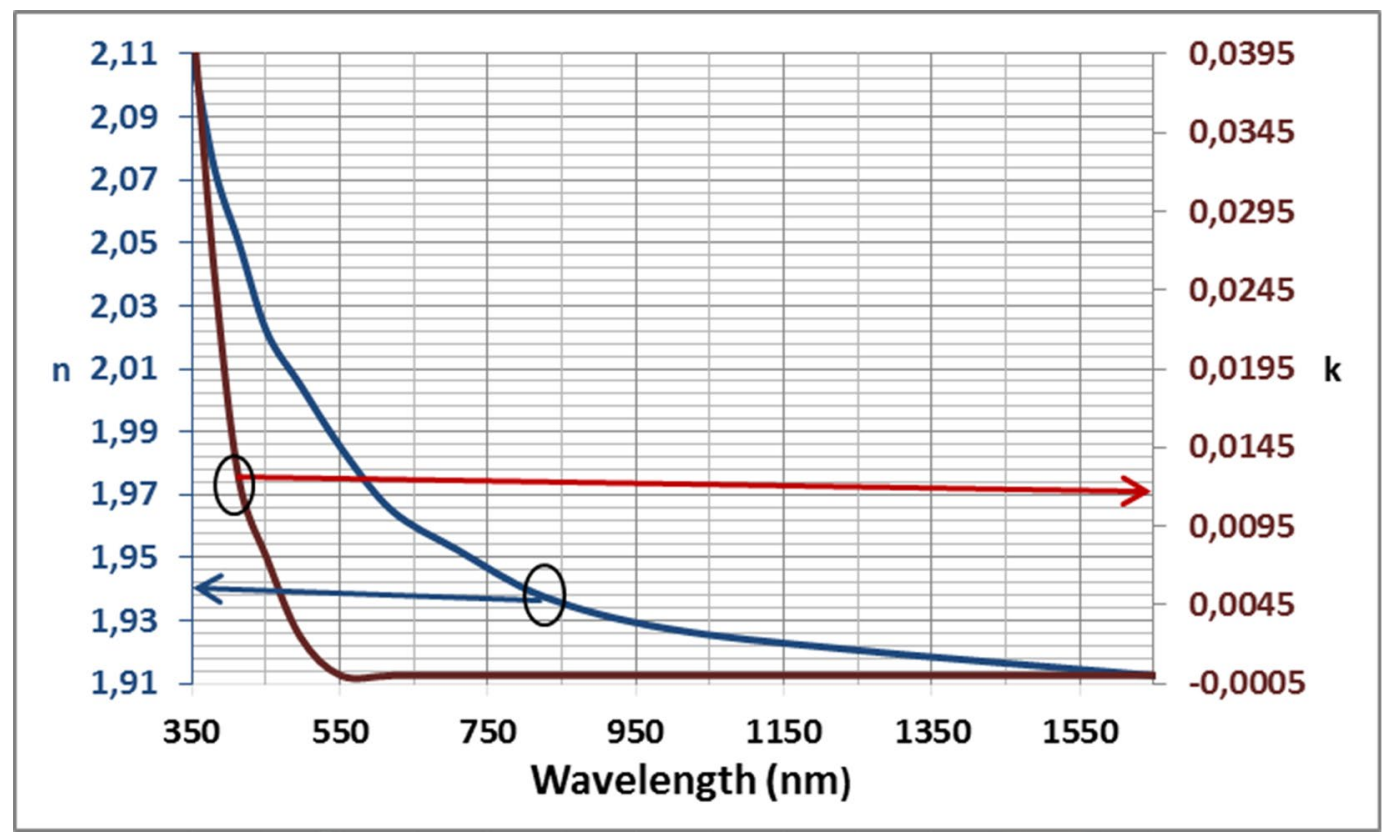

Fig. 2.- Refractive index of sputtered tin dioxide

\section{Experimental Set-up}

When the sensor was fabricated, the optical fiber was spliced to SMF pigtails connected to the light source and the optical spectrum analyzer (OSA). The light source HP-83437A was a superluminescent emitting diodes (SLED) white light source which has four LEDs at 1200, 1310, 1430 and $1550 \mathrm{~nm}$ and the OSA was the HP-86142A. The optical fiber was introduced in a climatic chamber (Angelantoni ACS CH 250), where the relative humidity has been increased from $20 \%$ to $90 \%$ at a constant temperature of $25{ }^{\circ} \mathrm{C}$. The experimental set-up is shown in Fig. 3.

Climatic chamber ACS CH250
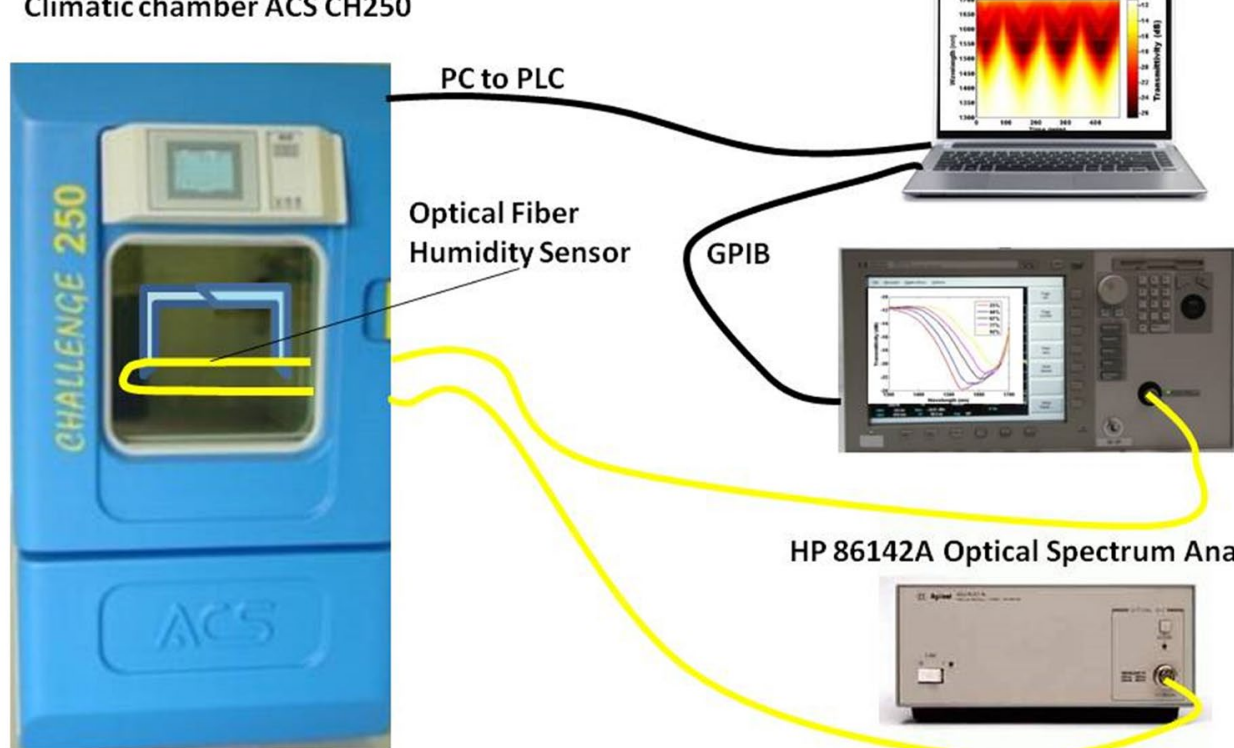

HP 86142A Optical Spectrum Analyzer

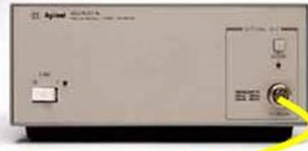

Agilent 83437A light source

Fig. 3.- Experimental set-up. 


\section{Simulations}

In this section the results obtained by simulations with FIMMWAVE ${ }^{\circledR}$ software are shown. Different parameters have been taken into account for these simulations. On one hand, we have simulated how the device would behave for different optical fiber diameters with the same thickness of tin oxide. Diameter of the optical fiber has been swept from $10 \mu \mathrm{m}$ to $30 \mu \mathrm{m}$ (core and cladding) while the tin oxide thickness was kept at $280 \mathrm{~nm}$. This diameter range has been chosen for a better understanding what role it plays on the performance of the device. Tin oxide thickness has been chosen for having a LMR in the OSA wavelength range. For simulating the effect of humidity on the thin film different layers of water have been added $(0$, 25 and $50 \mathrm{~nm}$ ). These theoretical results show that optical fiber diameter have not noticeable effect in the device sensitivity, but it has on the attenuation of the LMR. These results are shown in Fig. 4.
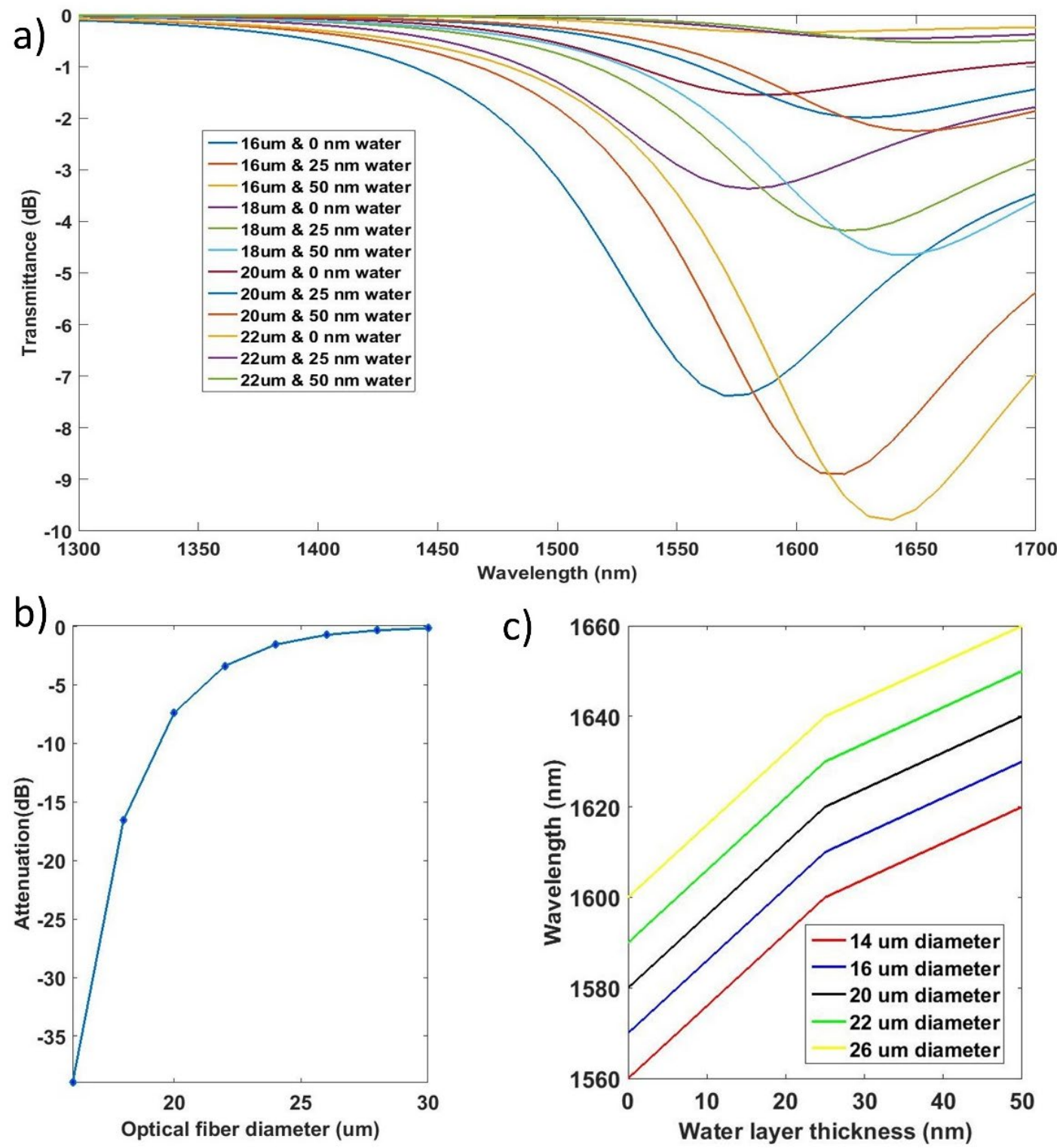

Fig.4.-a) Optical spectra of a CE-SMF with $280 \mathrm{~nm}$ of tin oxide for different diameters and different water layer thicknesses, b)attenuation of the LMR for different optical fiber diameters and c) wavelength position of the $L M R$ as a function of the water layer thickness for different diameters 
For diameters greater than $20 \mu \mathrm{m}$ the LMR is almost imperceptible and for diameters thinner than $16 \mu \mathrm{m}$ the attenuation exceeds $40 \mathrm{~dB}$ (Fig. 4 b)). In Fig. $4 \mathrm{c}$ ) it also can be observed than the sensitivity does not depend on the optical fiber diameter. Other interesting conclusion extracted from simulations is one related to coating thickness. Now variable thickness of tin oxide has been checked ( 140 to $300 \mathrm{~nm}$ ). Three different radius of the optical fiber (8.6, 9.6 and $10.6 \mathrm{um}$ ) have been used for simulating the effect of coating thickness on the sensitivity of the device. These results show that as the coating thickness increases the sensibility of the device decreases. However, the device has greater sensitivity for a given LMR when it is placed at greater wavelengths. Fig. 5 shows this theoretical results.

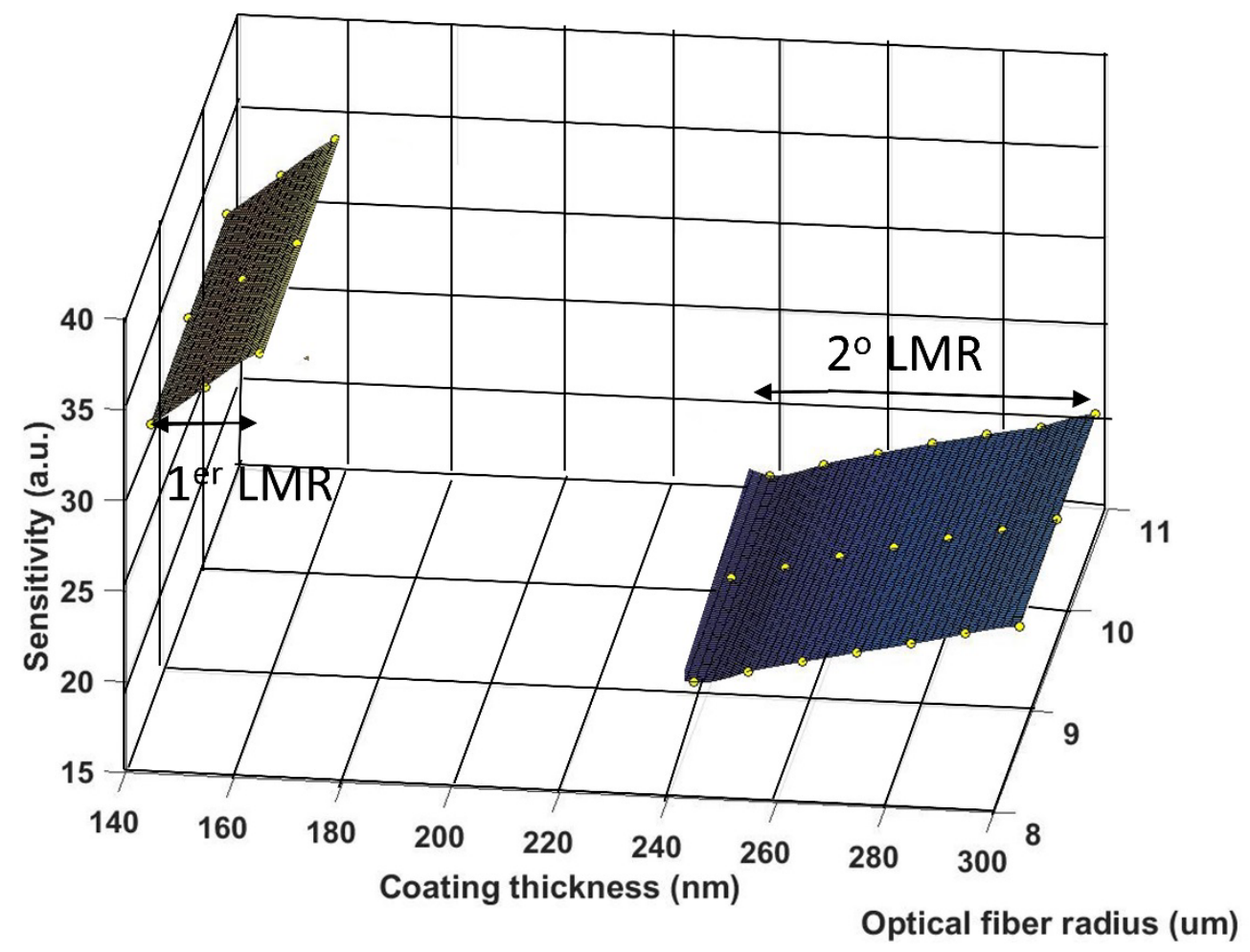

Fig.5.-Theoretical sensitivity for three different diameter of the CE-SMF, with variable coating thickness

Some design rules can be extracted from this simulations. Optical fiber diameter should be chosen regarding the wanted attenuation of the LMR. Optimum thickness of the coating depends on its refractive index, but maximum sensitivity of the device will be reach having the first LMR located at the greatest wavelength as possible, taking into account its displacement and the OSA range.

\section{Experimental Results}

\subsection{Humidity response}

The OFHS was introduced in a climatic chamber from Angelantoni Industries (ACS CH 250) where several cycles of $20 \%$ to $90 \%$ relative humidity $(\mathrm{RH})$ were applied while the temperature was keep constant to $25 \stackrel{\circ}{\circ}$. The slope was $1.16 \% \mathrm{RH} / \mathrm{min}$.

The construction was stopped when the first LMR was placed at a spectral position which was inside the range of the OSA. The position of the LMR wavelength varies as the refractive index of the external medium changes $[3,16]$ and by varying the relative humidity, the refractive index of the external medium as well as the refractive index of the $\mathrm{SnO}_{2}$ coating varies. The change of the refractive index of the coating is explained by the adsorbed water on the oxide surface [27, 
28] that occurs within the $\mathrm{SnO}_{2}$ structure. Characterization of the device has been made acquiring the relative humidity of the climatic chamber, by means of an electronic sensor, and collecting the optical spectrum at the same time. Then, the relationship between $\mathrm{RH}$ and the wavelength position of the LMR was established.

Changes on the spectrum have been collected at the same time that the relative humidity changed and they are shown in Fig. 6 . There is a wavelength shift of the position where the LMR occurs. The LMR shifts to greater wavelengths as the relative humidity increases, because of its sensitivity to the surrounding medium relative humidity. As it can be seen the device shows good repeatability and low hysteresis. A wavelength shift of $136 \mathrm{~nm}$ has been achieved for a relative humidity change from $20 \%$ to $90 \%$. The FWHM of the LMR is $93 \mathrm{~nm}$.
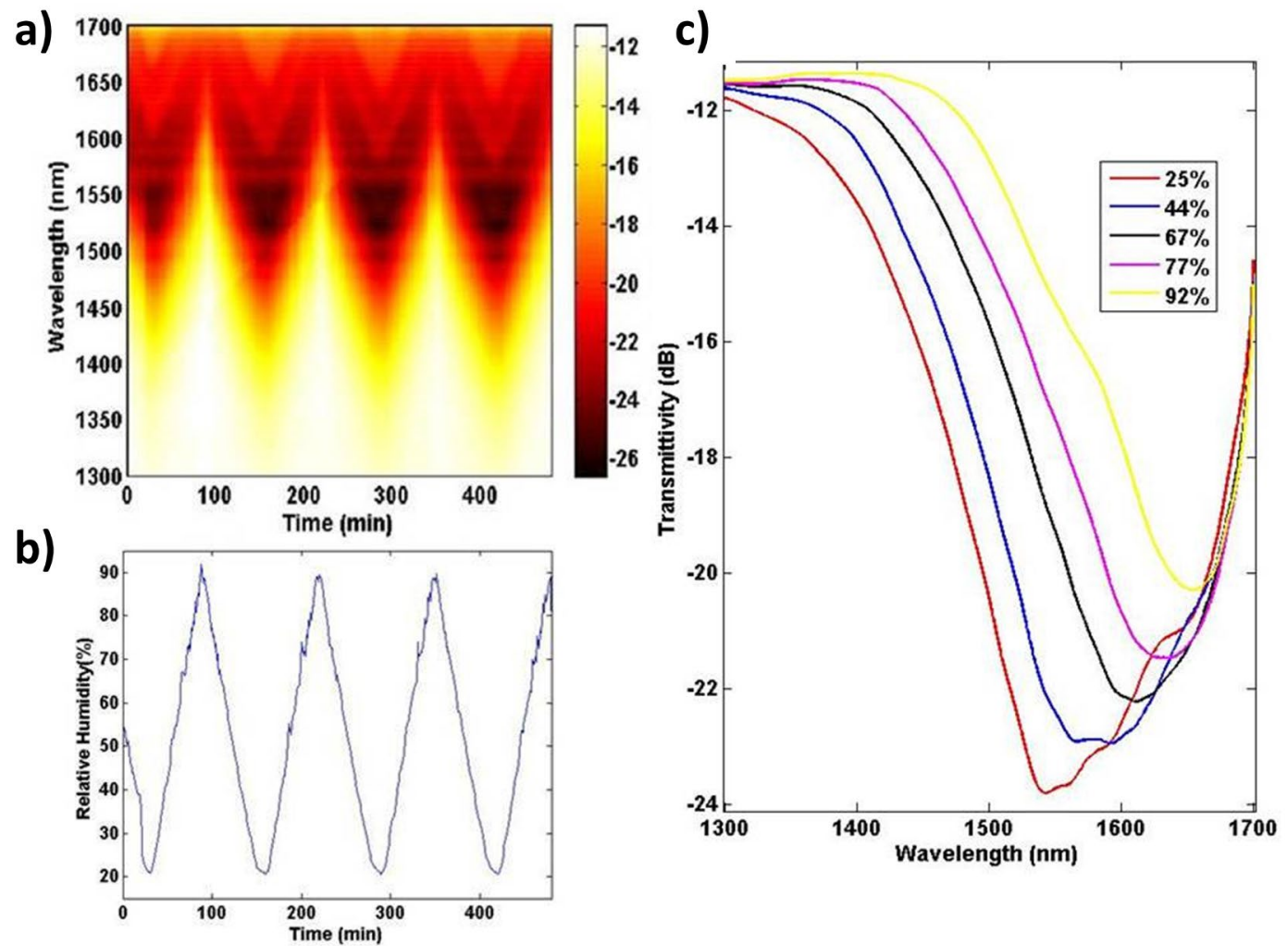

Fig. 6- a) Optical spectra for four cycles of $20 \%-90 \% \mathrm{RH}$, b) relative humidity cycles inside the climatic chamber and c) optical spectra for some values of $R H$

In Fig. 7, the LMR wavelength and the humidity are plotted as a function of time in the repetitive humidity cycles experiments. For the calculus of the LMR wavelength the spectra has been approximated by a quadratic fitting to a parabola and then, the wavelength position of the vortex has been located. These calculations were made using MATLAB ${ }^{\circledR}$ software. This graph shows how the LMR starts at $1525 \mathrm{~nm}$ at $21.5 \% \mathrm{RH}$, and how it reaches $1650 \mathrm{~nm}$ at $88.13 \% \mathrm{RH}$, achieving a total shift of $125 \mathrm{~nm}$. 


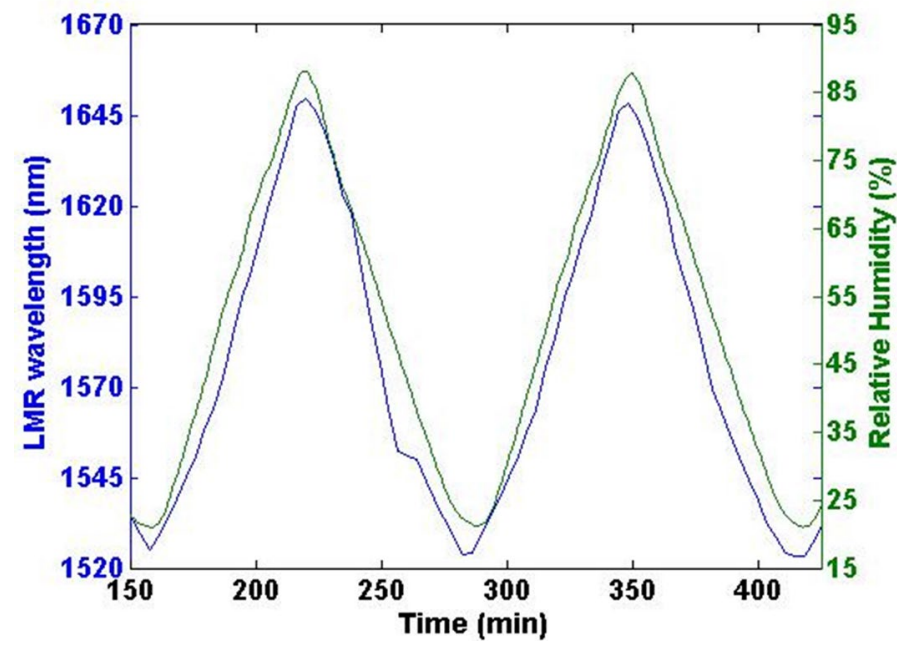

Fig. 7.- Representation of the LMR wavelength and the relative humidity as a function of time.

In Fig. 8 a), the characterization of the three OFHSs (D1, D3 \& D4) is plotted, which shows the relationship between relative humidity and wavelength shift. Device D1 shows low hysteresis; lower than $5 \% \mathrm{RH}$ in the full measurement range and almost zero for the $25-60 \% \mathrm{RH}$ range, and an average sensitivity of $1.9 \mathrm{~nm} / \% \mathrm{RH}$.
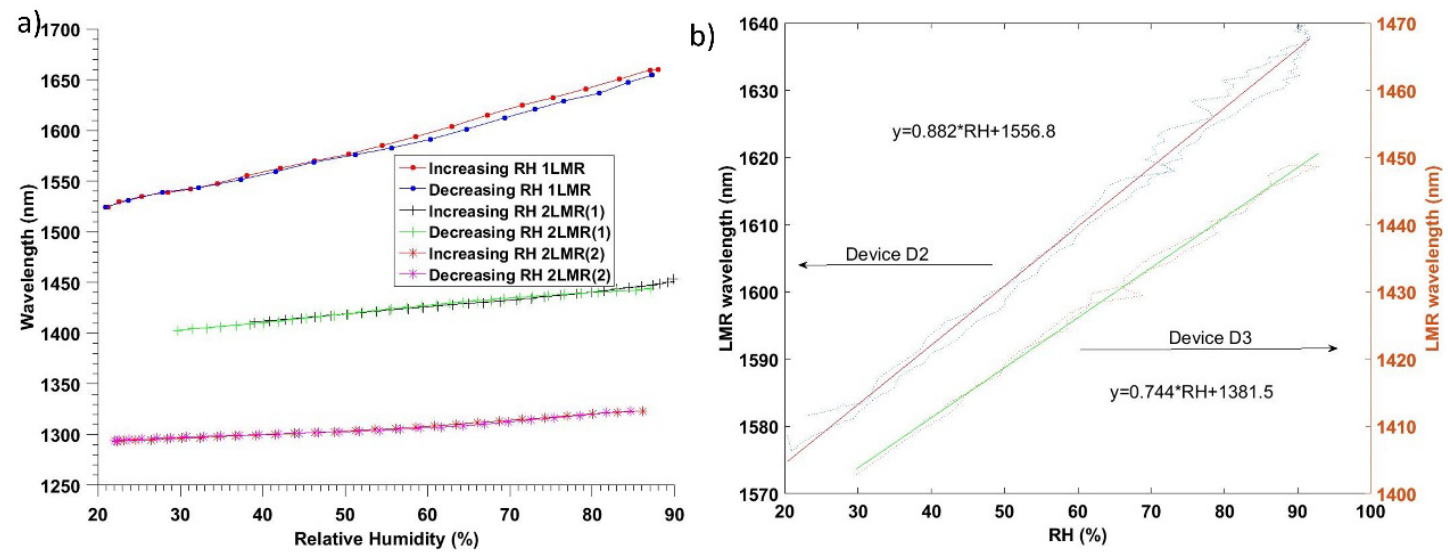

Fig. 8.- Representation of the $L M R$ wavelength as a function of the relative humidity.

For the device D3, with $280 \mathrm{~nm}$ of tin oxide coated onto the CE-SMF similar test was performed. As it has been shown in simulated results, the second LMR has lower sensitivity to relative humidity changes. Experimental results shown a sensitivity of $0.75 \mathrm{~nm} / \% \mathrm{RH}$, very low hysteresis and good linearity. Last device (D4) shows a sensitivity of $0.47 \mathrm{~nm} / \%$ RH. Finally, in Fig. $8 \mathrm{~b}$ ) it is shown how for the same LMR the sensitivity depends on the wavelength position of the LMR, obtaining a sensitivity of $0.882 \mathrm{~nm} / \% \mathrm{RH}$ for device $\mathrm{D} 2$, that has the second LMR located at 1570 $\mathrm{nm}$, improving the sensitivity of device D3, which has the same LMR located at $1400 \mathrm{~nm}$. These results are in good agreement with simulations and validate the model employed.

\subsection{Influence of temperature}

In order to obtain the cross thermal sensitivity of the device it has been used the same experimental set-up of Fig.3, but the relative humidity has kept constant while the temperature was changed from 20 to 50 으 in $5 \circ \mathrm{C}$ steps. Changes in temperature took 10 minutes while stabilization time was fixed at 30 minutes for the climatic chamber. This test has been performed for device D3 at 20\% RH and the results are shown in Fig. 9. As it can be seen, the LMR wavelength shifts $2 \mathrm{~nm}$ to lower wavelengths as the temperature increases from 20 to 50 ㅇ. . This response is nonlinear. Sensitivity to temperature is $0.066 \mathrm{~nm} /{ }^{\circ} \mathrm{C}$, and cross thermal sensitivity is $0.08 \% \mathrm{RH} /$ 으. 


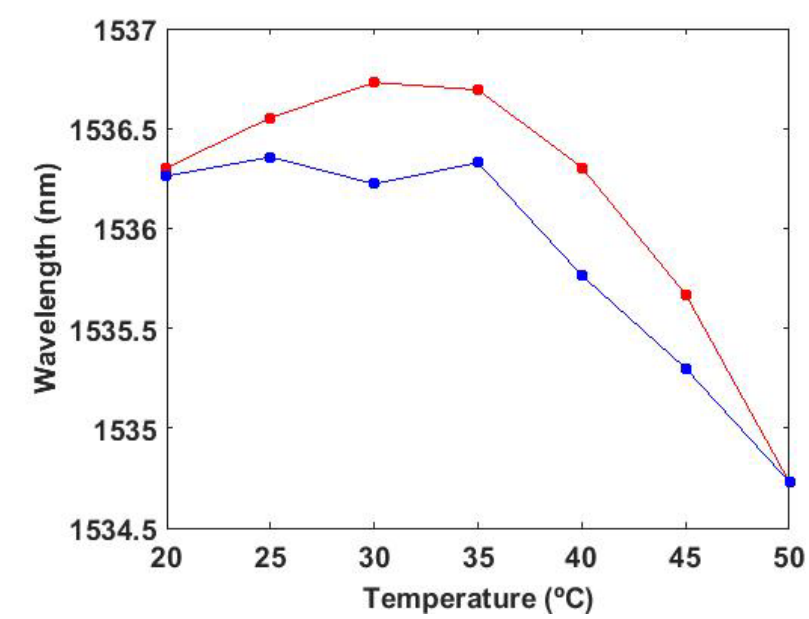

Fig. 9.- LMR wavelength position as a function of the temperature
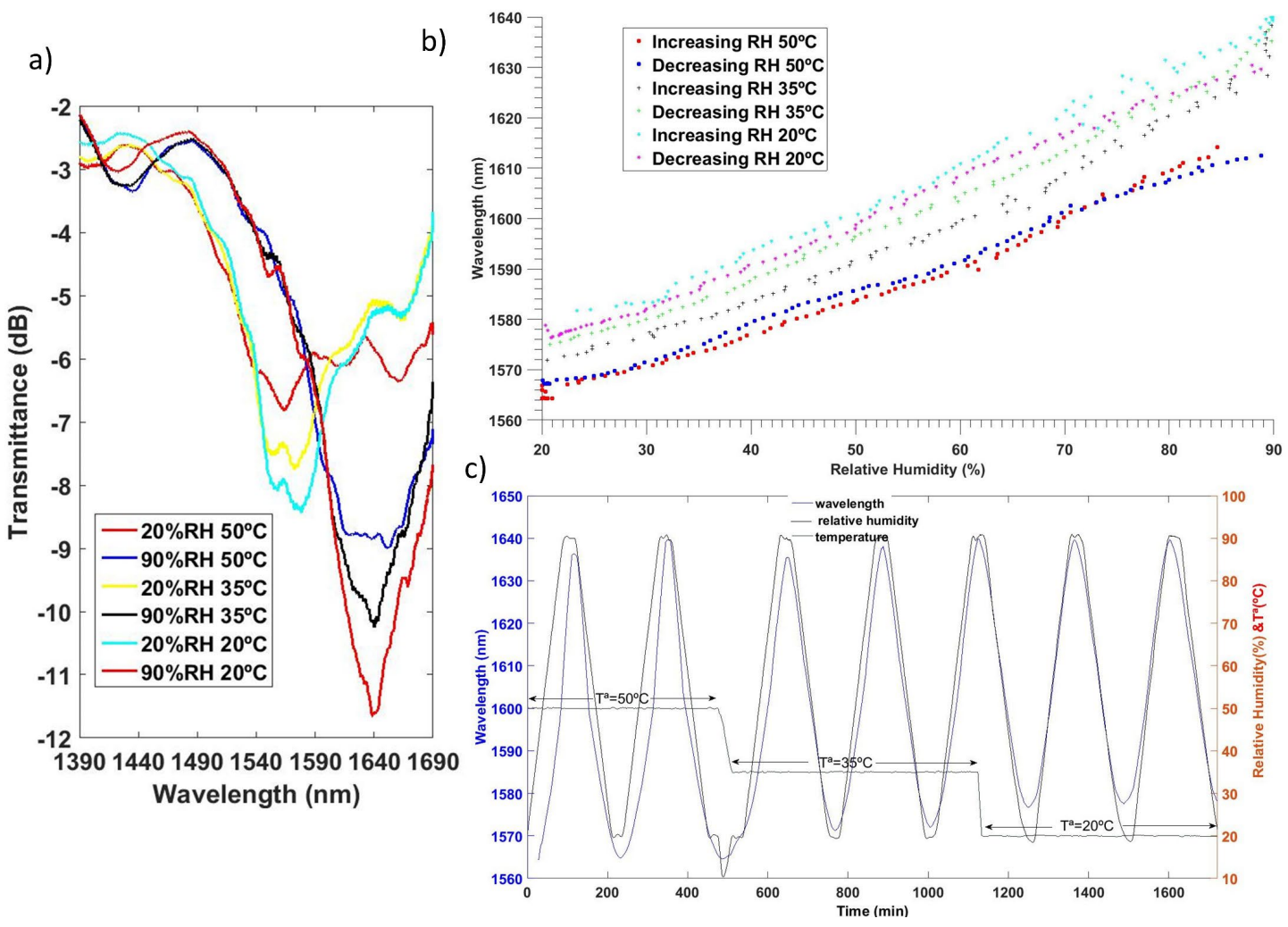

Fig. 10.-a) optical spectra for two different RHs and three temperatures, b) characterization of the LMR wavelength variations as a function of the relative humidity for three different temperatures and c) LMR wavelength variations following the climatic chamber.

Finally, another experiment was carried out to check how the temperature might affect to the sensitivity of the device. Another OFHS was subjected to 20-90 \%RH cycles at three different temperatures: 20,35 and $50^{\circ} \mathrm{C}$. Sensitivity of the device does not seem to be greatly influenced by temperature as it can be seen in Fig. $10 \mathrm{~b}$ ) and c), although there are changes on the initial wavelength position of the LMR. Temperature affects mainly to the attenuation of the LMR. Optical spectra for $20 \% \mathrm{RH}$ and $90 \% \mathrm{RH}$ at three different temperatures $(20,35$ and $50 \circ \mathrm{C})$ are shown in Fig. 10 a).

\subsection{Response time}


Finally, in order to see the response time of the device a $1550 \mathrm{~nm}$ laser (Rifocs) and a power meter (Rifocs $575 \mathrm{~L}$ ) have been used. It has been necessary to change the set-up for measuring the response time due to the large data acquisition time of the OSA and the relatively slow slope of the climatic chamber. Low flows of wet air were applied to the OFHS and the relative humidity was measured by an electronic humidity sensor (HIH 4000, Honeywell). Data were acquired by a data logger (Agilent 34972A). The experimental set-up for measuring the response time is shown in Fig. 11.

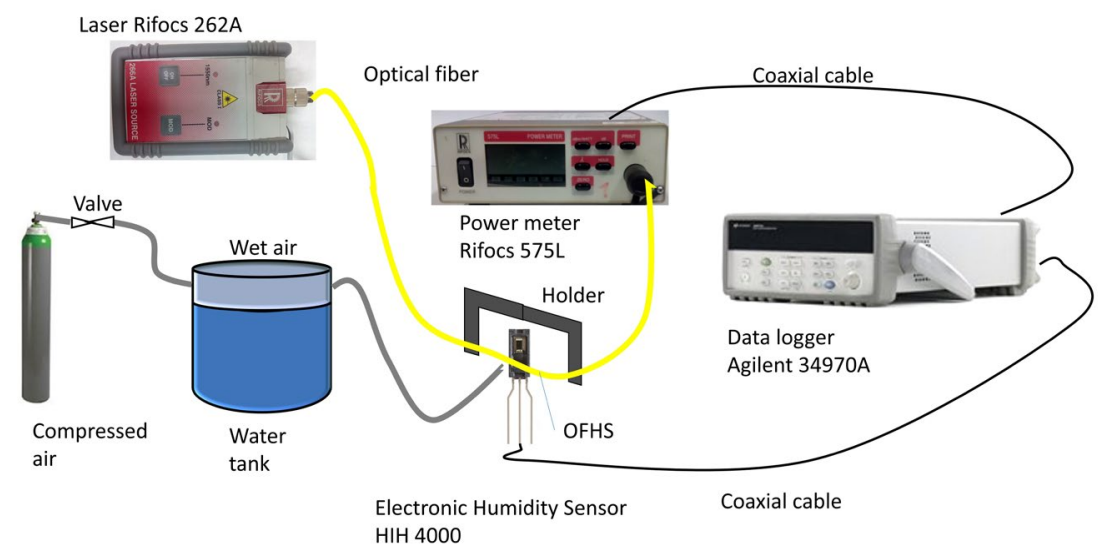

Fig. 11.- Experimental set-up for measuring the response time of the device.

By selecting a wavelength ( $1550 \mathrm{~nm}$ ) of the data acquired previously, it is possible to know the relationship between the optical power transmitted and the relative humidity. Changes in the optical power transmitted have been converted to changes in relative humidity, thanks to previous characterization of the device. The device presents a rise time of 1.52 second and similar fall time. The OFHS has smaller response times than the electronic humidity sensor, which is especially pronounced in the case of the fall time. Experimental data are shown in Fig. 12. Thickness has not noticeable effect on the response time for range studied in this paper. Parameters as maximum relative humidity, time of exposure or other parameters that have some influence on the roughness of the coating, as sputtering pressure [31], seems to have greater effect on the response time.
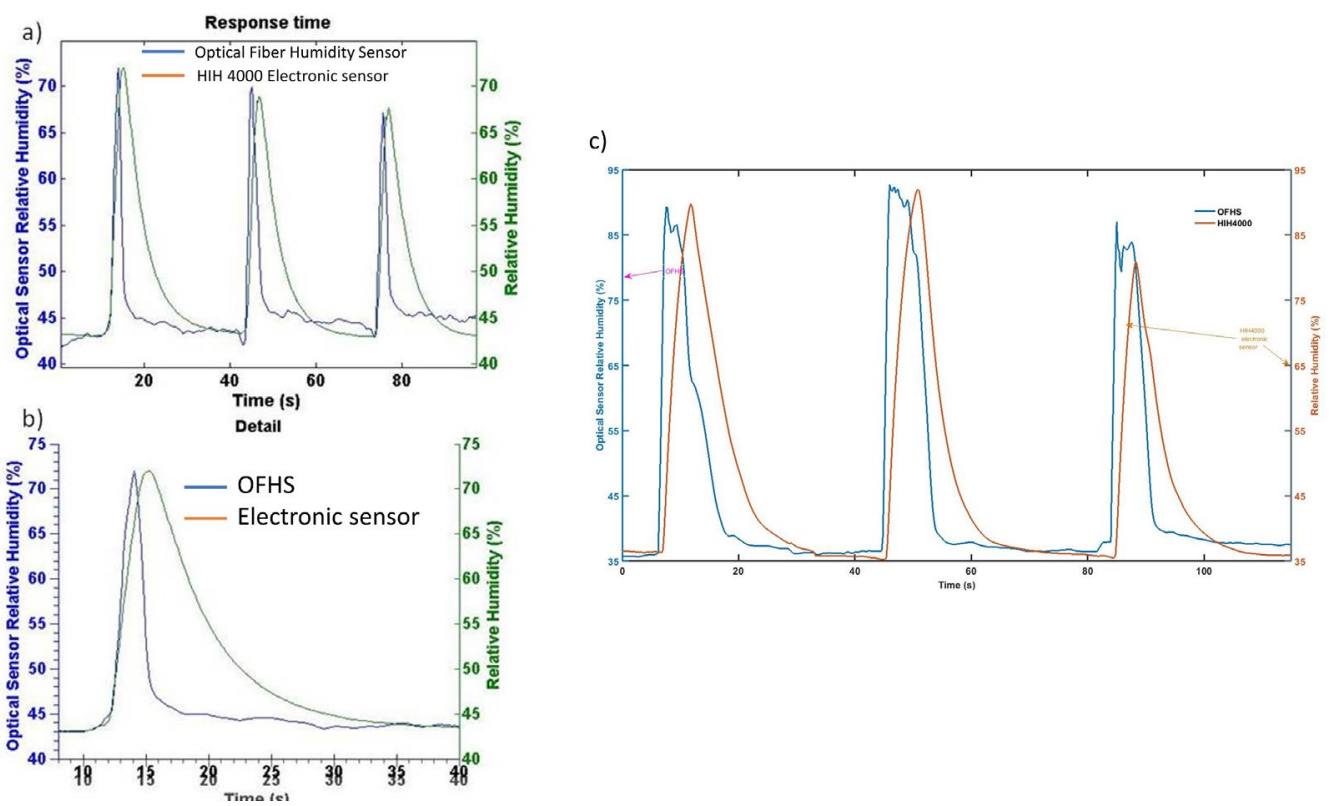

Fig. 12.- Response time of device $D 1$ a ffor three cycles, b) detail of the first cycle and c) response time of device D3, coated by DC sputtering.

\section{Conclusions}


A high sensitivity optical fiber humidity sensor has been fabricated by means of sputtering based on LMRs, by coating an etched single-mode optical fiber with a semiconductor material, tin oxide. Tin oxide has proved that it is a good material for LMR generation and for humidity sensing. This optical structure can be used for multiple sensing purposes, just choosing the appropriate material which meets the requirements for LMR generation and that is sensitive to the variable of interest, like biomedical sensors [14], pH sensors [19], refractometers [13], electro-optical devices [15], etc.

Optical fiber diameter has not influence on the sensitivity, but it has on the attenuation of the LMR. A minimum diameter of $30 \mu \mathrm{m}$ is needed for obtaining the LMR, although the attenuation band is smaller than $0.3 \mathrm{~dB}$, which may difficult the detection of the peak. Greater sensitivities have been obtained for the first LMR (140 nm of tin oxide coating), and the greatest when the first LMR is placed near $1550 \mathrm{~nm}$. Sensitivity decreases with the order of the LMR, but it increases with the wavelength where the LMR is placed for a given LMR. Temperature might affect the attenuation of the LMR and the initial wavelength of the LMR but it does not have noticeable effect on sensitivity.

Maximum wavelength shifts of $130 \mathrm{~nm}$ have been achieved for relative humidity values varying from $20 \%$ to $90 \%$, which implies a sensitivity of $1.9 \mathrm{~nm} / \% \mathrm{RH}$. The OFHS shows good repeatability, low hysteresis, and also it presents larger dynamic range and better sensitivity, $35 \%$ greater than the better found based on LMRs [17] and $200 \%$ greater than other devices $[8,18,28]$.

\section{Acknowledgment}

This work was supported by a Public University of Navarra PhD Grant and by the Spanish Economy and Competitiveness Ministry TEC-2013-43679-R Research Grant.

\section{References}

[1] Gastón, A., Pérez, F., \& Sevilla, J. (2004). Optical fiber relative-humidity sensor with polyvinyl alcohol film. Applied optics, 43(21), 4127-4132

[2] Bariain, C., Matías, I. R., Arregui, F. J., \& Lopez-Amo, M. (2000). Optical fiber humidity sensor based on a tapered fiber coated with agarose gel. Sensors and Actuators B: Chemical, 69(1), 127-131.

[3] Harith, Z., Irawati, N., Rafaie, H. A., Batumalay, M., Harun, S. W., Nor, R. M., \& Ahmad, H. (2015). Tapered Plastic Optical Fiber Coated With Al-Doped ZnO Nanostructures for Detecting Relative Humidity. Sensors Journal, IEEE, 15(2), 845-849.

[4] Batumalay, M., Ahmad, F., Arof, H., Ahmad, H., \& Harun, S. W. (2013). Tapered plastic optical fiber coated with HEC/PVDF for measurement of relative humidity. Sensors Journal, IEEE, 13(12), 4702-4705.

[5] Hernaez, M., Zamarreño, C. R., Fernandez-Valdivielso, C., Del Villar, I., Arregui, F. J., \& Matias, I. R. (2010). Agarose optical fibre humidity sensor based on electromagnetic resonance in the infrared region. physica status solidi (c), 7(11-12), 2767-2769.

[6] Konstantaki, M., Pissadakis, S., Pispas, S., Madamopoulos, N., \& Vainos, N. A. (2006). Optical fiber long-period grating humidity sensor with poly (ethylene oxide)/cobalt chloride coating. Applied optics, 45(19), 4567-4571.

[7] Zheng, S., Zhu, Y., \& Krishnaswamy, S. (2013). Fiber humidity sensors with high sensitivity and selectivity based on interior nanofilm-coated photonic crystal fiber long-period gratings. Sensors and Actuators B: Chemical, 176, 264-274.

[8] Hao Sun, Zaihang Yang, Libin Zhou, Nan Liu, Tingting Gang, Xueguang Qiao, Manli Hu, A relative humidity sensing probe based on etched thin-core fiber coated with polyvinyl alcohol, Optics Communications, Volume 356, 1 December 2015, Pages 556-559, 
[9] Ignacio Del Villar, Abian B. Socorro, Miguel Hernaez, et al., "Sensors Based on Thin-Film Coated Cladding Removed Multimode Optical Fiber and Single-Mode Multimode SingleMode Fiber: A Comparative Study", Journal of Sensors, vol 2015, Article ID 763762

[10] Arregui, F. J., Del Villar, I., Corres, J. M., Goicoechea, J., Zamarreño, C. R., Elosua, C., ... \& Matias, I. R. (2014). Fiber-optic Lossy Mode Resonance Sensors. Procedia Engineering, 87, 3-8.

[11] I. Del Villar, C. R. Zamarreño, M. Hernaez, F. J. Arregui, and I. R. Matias, "Lossy mode resonance generation with indium-tin-oxide coated optical fibers for sensing applications," J. Lightw. Technol., vol. 28, no. 1, pp. 111-117, Jan. 2010.

[12] Socorro, A. B., Del Villar, I., Corres, J. M., Arregui, F. J., \& Matias, I. R. (2012). Tapered singlemode optical fiber $\mathrm{pH}$ sensor based on lossy mode resonances generated by a polymeric thin-film. Sensors Journal, IEEE, 12(8), 2598-2603.

[13] Hernáez, M., Del Villar, I., Zamarreño, C. R., Arregui, F. J., \& Matias, I. R. (2010). Optical fiber refractometers based on lossy mode resonances supported by $\mathrm{TiO}_{2}$ coatings. Applied optics, 49(20), 3980-3985.

[14] Razquin, L., Zamarreno, C. R., Munoz, F. J., Matias, I. R., \& Arregui, F. J. (2012, October). Thrombin detection by means of an aptamer based sensitive coating fabricated onto LMRbased optical fiber refractometer. In Sensors, 2012 IEEE (pp. 1-4). IEEE.

[15] Corres, J. M., Ascorbe, J., Arregui, F. J., \& Matias, I. R. (2013). Tunable electro-optic wavelength filter based on lossy-guided mode resonances. Optics express, 21(25), 3166831677.

[16] Hernaez, M., Zamarreño, C. R., Del Villar, I., Arregui, F. J., \& Matias, I. R. (2009). Optical fiber humidity sensor based on lossy mode resonances.International journal on smart sensing and intelligent systems, 2(4), 653-660.

[17] Zamarreño, C. R., Hernaez, M., Sanchez, P., Del Villar, I., Matias, I. R., \& Arregui, F. J. (2011). Optical fiber humidity sensor based on lossy mode resonances supported by TiO2/PSS coatings. Procedia Engineering, 25, 1385-1388.

[18] Ascorbe J., Corres J.M., Arregui F.J., Matías I.R. Optical fiber humidity sensor based on tapered fiber asymmetrically coated with indium tin oxide, IEEE Sensors 2014, pp 1916-1919

[19] Socorro, A. B., Del Villar, I., Corres, J. M., Arregui, F. J., \& Matias, I. R. (2012). Tapered singlemode optical fiber $\mathrm{pH}$ sensor based on lossy mode resonances generated by a polymeric thin-film. Sensors Journal, IEEE, 12(8), 2598-2603.

[20] Haddock, H. S., Shankar, P. M., \& Mutharasan, R. (2003). Fabrication of biconical tapered optical fibers using hydrofluoric acid. Materials Science and Engineering: B, 97(1), 87-93.

[21] Kbashi, H. J. (2012). Fabrication of submicron-diameter and taper fibers using chemical etching. Journal of Materials Science \& Technology, 28(4), 308-312.

[22] Corres, J. M., Arregui, F. J., \& Matias, I. R. (2006). Design of humidity sensors based on tapered optical fibers. Lightwave Technology, Journal of, 24(11), 4329-4336.

[23] Jiao, L. Z., Dong, D. M., Zheng, W. G., Wu, W. B., Shen, C. J., \& Yan, H. (2013). Research on fiber-optic etching method for evanescent wave sensors. Optik-International Journal for Light and Electron Optics, 124(8), 740-743.

[24] Linslal, C. L., Mohan, P. S., Halder, A., \& Gangopadhyay, T. K. (2013). Analysis and modeling of an optical fiber loop resonator and an evanescent field absorption sensor for the application for chemical detection. Sensors and Actuators A: Physical, 194, 160-168.

[25] Mysliwieca, M., Grochowskia, J., Krogulskia, K., Mikulicb, P., \& Bockb, W. J. (2013). Effect of Wet Etching of Arc-Induced Long-Period Gratings on Their Refractive Index Sensitivity. 
[26] Zamarreño, C. R., Hernaez, M., Del Villar, I., Matias, I. R., \& Arregui, F. J. (2011, October). Lossy mode resonance-based optical fiber humidity sensor. In Sensors, 2011 IEEE (pp. 234237). IEEE.

[27] Sanchez, P., Zamarreño, C. R., Hernaez, M., Del Villar, I., Fernandez-Valdivielso, C., Matias, I. R., \& Arregui, F. J. (2012). Lossy mode resonances toward the fabrication of optical fiber humidity sensors. Measurement Science and Technology, 23(1), 014002.

[28] Sanchez, P., Zamarreño, C. R., Hernaez, M., del Villar, I., Matias, I. R., \& Arregui, F. J. (2013, May). Humidity sensor fabricated by deposition of SnO2 layers onto optical fibers. In Fifth European Workshop on Optical Fibre Sensors (pp. 87940C-87940C). International Society for Optics and Photonics.

[29] Faia, P. M., \& Furtado, C. S. (2013). Effect of composition on electrical response to humidity of TiO 2: $\mathrm{ZnO}$ sensors investigated by impedance spectroscopy. Sensors and Actuators B: Chemical, 181, 720-729.

[30] Gercher, V. A., \& Cox, D. F. (1995). Water adsorption on stoichiometric and defective SnO 2 (110) surfaces. Surface science, 322(1), 177-184.

[31] Jung, Y. S., Lee, D. W., \& Jeon, D. Y. (2004). Influence of dc magnetron sputtering parameters on surface morphology of indium tin oxide thin films. Applied surface science, 221(1), 136142.

\section{Figure Captions}

Fig 1. Cladding-etched single mode optical fiber.

Fig 2. Refractive index of sputtered tin dioxide.

Fig 3. Experimental set-up.

Fig 4. a) Optical spectra for four cycles of $20 \%-90 \% \mathrm{RH}$, b) relative humidity cycles inside the climatic chamber and c) optical spectra for some values of $\mathrm{RH}$.

Fig 5. Representation of the LMR wavelength and the relative humidity as a function of time.

Fig 6. Representation of the $L M R$ wavelength as a function of the relative humidity.

Fig 7. Experimental set-up for measuring the response time of the device.

Fig 8. Response time of the device a) for three cycles and b) detail of the first cycle. 